\title{
South Italy: A Privileged Perspective to Understand the Relationship between Hidradenitis Suppurativa and Overweight/ Obesity
}

\author{
Gabriella Fabbrocini Valerio De Vita Marianna Donnarumma Giuseppe Russo \\ Giuseppe Monfrecola
}

Division of Clinical Dermatology, Department of Clinical Medicine and Surgery, University of Naples Federico II, Naples, Italy

\section{Key Words}

Hidradenitis · Obesity · Childhood

\begin{abstract}
Background: Hidradenitis suppurativa (HS) is a chronic inflammatory skin condition that involves the terminal hair follicles of apocrine gland-bearing skin areas. Objective: To evaluate the relationship between $\mathrm{HS}$ and systemic medical conditions, such as overweight/obesity during adolescence. Methods: Epidemiologic data was collected from January 2014 up to December 2015 from 27 patients affected by HS, outpatients of the Dermatology Department of University of Naples Federico II, to examine the correlation between HS and overweight/obesity. Results: $70.4 \%$ of the patients were female and $29.6 \%$ were male. The mean age at onset of the disease was $18.1 \pm 5.99$ years, while the mean age at first diagnosis by the physician was $21.77 \pm 6.61$ years. A mean Sartorius score of $50.26 \pm 21.75$ was registered. Body mass index of HS patients was positively correlated with Sartorius score. Conclusion: Our study underlines the fact that particular attention to metabolic alteration in adolescence can lead to a correct diagnosis with less delay in HS.
\end{abstract}

\section{Introduction}

Hidradenitis suppurativa (HS) is a chronic inflammatory skin condition that involves the terminal hair follicles of apocrine gland-bearing skin areas, characterized by painful nodules, recurrent abscesses, draining sinuses and scarring tracts. HS affects mainly women, in particular young adults. HS is a multifactorial pathological entity in which genetic and/or environmental factors combine in the pathogenesis of the disease. In recent times, HS has been considered as a systemic disease because of the possible association with cardiometabolic comorbidities and other conditions [1-3].

\section{Methods}

From January 2014 to December 2015, 27 patients with HS among the outpatients of the Dermatology Department of University of Naples Federico II were involved in the study (table 1). Written informed consent was signed by the patients or by their parents for subjects younger than 18 years.

The collected information included demographic data, anthropometric measures, clinical history of the disease, clinical features at entry and follow-up, presence of comorbidities, blood tests, including blood glucose and insulin levels, by which it was

\section{KARGER}

E-Mail karger@karger.com

www.karger.com/sad (c) 2016 S. Karger AG, Base

2296-9195/16/0022-0052\$39.50/0
Prof. Gabriella Fabbrocini, MD, Division of Clinical Dermatology

Department of Clinical Medicine and Surgery

University of Naples Federico II, via S. Pansini 5

IT-80131 Naples (Italy)

E-Mail gafabbro@unina.it 
Table 1. Demographics, anthropometric variables, smoking, and baseline clinical features of patients included in our sample

\begin{tabular}{|c|c|c|c|c|c|c|c|}
\hline & \multicolumn{2}{|c|}{ Overall } & \multicolumn{2}{|c|}{ Male } & \multicolumn{2}{|c|}{ Female } & \multirow[t]{2}{*}{$\mathrm{p}$ value } \\
\hline & $\mathrm{n}$ & $\%$ & $\mathrm{n}$ & $\%$ & $\mathrm{n}$ & $\%$ & \\
\hline \multicolumn{8}{|l|}{ Epidemiological data } \\
\hline Patients & 27 & 100.0 & 8 & 29.6 & 19 & 70.4 & \\
\hline Age, years $($ mean $\pm S D)$ & \multicolumn{2}{|c|}{$33.74 \pm 7.29$} & \multicolumn{2}{|c|}{$35.87 \pm 4.01$} & \multicolumn{2}{|c|}{$32.84 \pm 8.22$} & 0.56 \\
\hline$<20$ & 2 & 7.4 & 0 & 0 & 2 & 10.5 & \\
\hline $20-29$ & 5 & 18.6 & 1 & 12.5 & 4 & 21 & \\
\hline $30-39$ & 11 & 40.7 & 5 & 62.5 & 6 & 31.7 & \\
\hline$\geq 40$ & 9 & 33.3 & 2 & 25 & 7 & 36.8 & \\
\hline \multicolumn{8}{|l|}{ Age at first diagnosis by } \\
\hline physician, years (mean $\pm S D$ ) & \multicolumn{2}{|c|}{$21.77 \pm 6.61$} & \multicolumn{2}{|c|}{$26.125 \pm 5.59$} & \multicolumn{2}{|c|}{$20.21 \pm 4.37$} & 0.018 \\
\hline$<20$ & 0 & 0 & 0 & 0 & 0 & 0 & \\
\hline $20-29$ & 9 & 33.3 & 0 & 0 & 9 & 47.35 & \\
\hline $30-39$ & 14 & 51.9 & 5 & 62.2 & 9 & 47.35 & \\
\hline$\geq 40$ & 4 & 14.8 & 3 & 37.5 & 1 & 5.3 & \\
\hline Age at onset, years (mean $\pm S D)$ & \multicolumn{2}{|c|}{$18.1 \pm 5.99$} & \multicolumn{2}{|c|}{$22.12 \pm 5.56$} & \multicolumn{2}{|c|}{$16.52 \pm 5.47$} & 0.01 \\
\hline$<10$ & 2 & 7.4 & 0 & 0 & 2 & 10.5 & \\
\hline $10-19$ & 17 & 63 & 3 & 37.5 & 14 & 73.7 & \\
\hline $20-29$ & 6 & 22.2 & 4 & 50 & 2 & 10.5 & \\
\hline$\geq 30$ & 2 & 7.4 & 1 & 12.5 & 1 & 5.3 & \\
\hline \multicolumn{8}{|l|}{ Metabolic data } \\
\hline BMI (mean \pm SD) & \multicolumn{2}{|c|}{$28.79 \pm 6.03$} & \multicolumn{2}{|c|}{$27.99 \pm 2.14$} & \multicolumn{2}{|c|}{$29.13 \pm 7.09$} & 0.94 \\
\hline $20.0-24.9$ & 5 & 18.6 & 1 & 12.5 & 4 & 21 & \\
\hline $25.0-29.9$ & 15 & 55.4 & 5 & 62.5 & 10 & 52.7 & \\
\hline$\geq 30.0$ & 7 & 26 & 2 & 25 & 5 & 26.3 & \\
\hline Smoking habit & & & & & & & 0.4 \\
\hline Yes & 14 & 51.8 & 3 & 37.5 & 11 & 58 & \\
\hline No & 13 & 48.2 & 5 & 62.5 & 8 & 42 & \\
\hline \multicolumn{8}{|l|}{ Clinical data } \\
\hline Sartorius score (mean $\pm \mathrm{SD}$ ) & \multicolumn{2}{|c|}{$50.26 \pm 21.75$} & \multicolumn{2}{|c|}{$49.75 \pm 14.4$} & \multicolumn{2}{|c|}{$50.47 \pm 24.55$} & 0.92 \\
\hline$<25$ & 4 & 14.8 & 0 & 0 & 4 & 21 & \\
\hline $25-49$ & 7 & 26 & 2 & 25 & 5 & 26.3 & \\
\hline $50-75$ & 10 & 37 & 5 & 62.5 & 5 & 26.3 & \\
\hline$>75$ & 6 & 22.2 & 1 & 12.5 & 5 & 26.3 & \\
\hline \multicolumn{8}{|l|}{ Main affected locations } \\
\hline Groin/genitals & 11 & 40.7 & 3 & 37.5 & 8 & 42.1 & \\
\hline Axillae & 18 & 66.6 & 5 & 62.5 & 13 & 68.4 & \\
\hline Buttocks & 4 & 14.8 & 1 & 12.5 & 3 & 15.9 & \\
\hline Back and chest & 1 & 3.7 & 1 & 12.5 & 0 & 0 & \\
\hline Submammary region & 5 & 18.6 & 0 & 0 & 5 & 26.3 & \\
\hline
\end{tabular}

possible to calculate the homoeostasis model assessment of insulin resistance (HOMA-IR). Severity of HS was assessed by the physician using the Sartorius score. The Sartorius score is a method to quantify the severity of HS through a scale of numerical scores. Points are awarded for (1) anatomic region involved (axilla, groin, genital, gluteal, or other inflammatory region left and/or right): 3 points per each body area involved; (2) number and type of lesions (abscesses, nodules, fistulas, scars): 2 points for each nodule, 4 points for each fistula, 1 point for each scar, 1 point each for 'other'; (3) longest distance between two relevant lesions (i.e., nodules and fistulas, in each region): 2 points for less than $5 \mathrm{~cm}, 4$ points for less than $10 \mathrm{~cm}, 8$ points for more than $10 \mathrm{~cm}$; or lesion size if only one lesion present: 2 points for less than $5 \mathrm{~cm}, 4$ points for less than $10 \mathrm{~cm}, 8$ points for more than $10 \mathrm{~cm}$; (4) lesions clearly separated by normal skin in each region: 0 points for 'yes', 6 points for 'no'. Information about the age of onset, the age of first diagnosis, body mass index (BMI), age, smoking habits, Sartorius score and locations involved was collected. Correlation analysis was conducted between the Sartorius score and BMI in all patients, and the Sartorius score and BMI in both sexes. The correlation between disease severity and duration was also evaluated. 


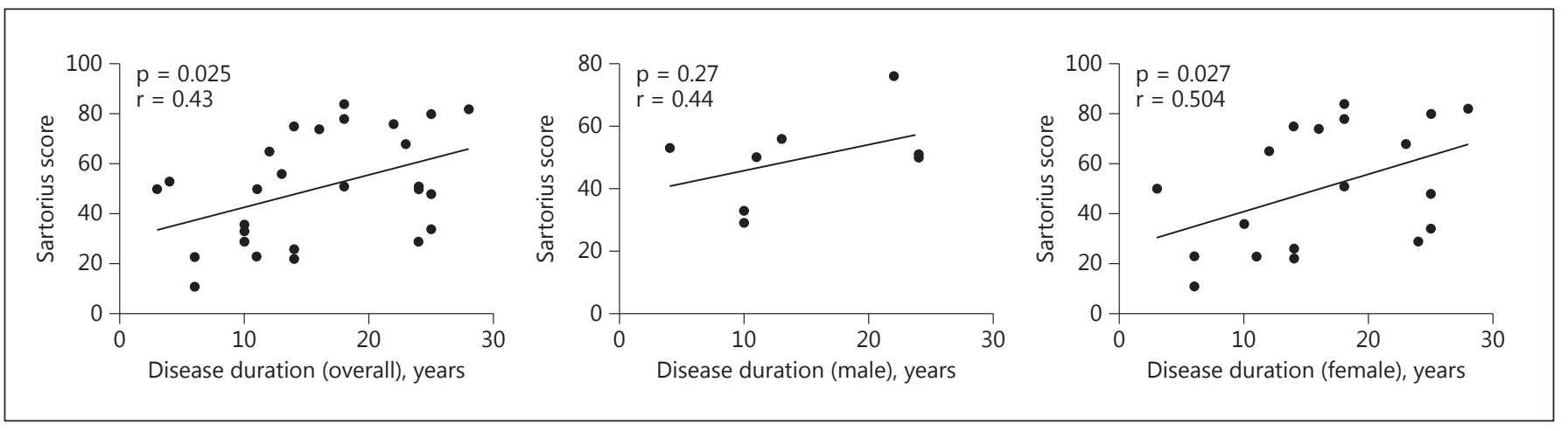

Fig. 1. Correlation between the severity (Sartorius score) and the duration of the disease.

All statistical analyses were performed using GraphPad Prism 4.0 (GraphPad Software Inc., La Jolla, Calif., USA). Mann-Whitney test was used to calculate statistical differences and the values of p correlations were evaluated with Spearman's rho test (table 1).

\section{Results}

$70.4 \%$ of the patients were female and $29.6 \%$ were male. The mean age at onset of the disease was $18.1 \pm 5.99$ years. A later age of onset was detected in males $(22.12$ years), showing a statistically significant difference compared to females (16.52 years) $(\mathrm{p}=0.01)$.

The mean age at first diagnosis by the physician was $21.77 \pm 6.6$ years. A later age of diagnosis was detected in males (26.12 years), compared to females (20.12 years) $(\mathrm{p}=0.01)$.

In the population of the Italian Registry for Hidradenitis Suppurativa (IRHS) [4], HS seems to arise less early in life $(33.4 \pm 13.0$ years $)$ and is diagnosed with a bigger delay $(28.2 \pm 11.8$ years $)$. Our sample shows a statistically significant variation $(\mathrm{p}<0.0001)$ in the age of onset in comparison with the data of the Italian registry. This data may correlate with a higher percentage of overweight and obesity in childhood in our sample. Interestingly, HS patients presented a higher BMI and HOMA-IR. The BMI is defined as the body weight divided by the square of the body height, and is universally expressed in units of $\mathrm{kg} /$ $\mathrm{m}^{2}$, resulting from weight in kilograms and height in meters. BMI is used to categorize people as underweight, normal weight, overweight, or obese. Commonly accepted BMI ranges are underweight: under 18.5; normal weight: 18.5-25; overweight: 25-30; obese: over 30. In our sample, the BMI was $28.79 \pm 6.03$ versus $26.3 \pm 5.5$ of the
IRHS population. $81 \%$ of our patients had a BMI higher than 25 versus $51.5 \%$ of the IRHS patients.

The severity of the disease was calculated by Sartorius score and a mean Sartorius score of $50.26 \pm 21.75$ was registered. The main affected locations at baseline were axillae $(66.6 \%)$, groin/genitals (40.7\%), buttocks $(14.8 \%)$, trunk $(3.7 \%)$ and submammary region $(18.6 \%)$, while Italian national data describes a Sartorius score of $78.4 \pm$ 66.1 with the following distribution: groin/genitals (44.1\%), axillae (34.7\%), buttocks (10.2\%) and trunk (9.0\%). The severity of the disease, measured by the Sartorius score, correlates positively with BMI. A statistically significant positive correlation between the severity (Sartorius score) and the duration of the disease has been demonstrated ( $\mathrm{p}=0.025)$ (fig. 1).

In our sample approximately $51.8 \%$ of the patients were current smokers, with a significantly higher percentage compared to the Italian population [5]. The most frequently reported comorbidities were acne $(7.9 \%)$, obesity $(5.1 \%)$, diabetes $(4.2 \%)$ and thyroid diseases $(3.6 \%)$. The data is similar to the data of the Italian registry.

\section{Discussion and Conclusion}

The early onset of HS in our region and its association with diabetes are not surprising, as the Campania region faces an overweight/obesity epidemic affecting both children and adults, with the highest prevalence compared to other regions of Italy and Europe. According to the 2014 annual report of the National Observatory of Health in Italian Regions, among the adult population of the region Campania, $10.3 \%$ are affected by obesity and $41.6 \%$ are in an overweight condition. Moreover, in our region, the
Fabbrocini/De Vita/Donnarumma/Russo/ Monfrecola 
prevalence of obesity among children and adolescents is among the highest in Europe. The high prevalence of obesity in the pediatric population could possibly explain why HS arises earlier in Campania with respect to the rest of Italy: $38.9 \%$ of the population aged between 6 and 17 is overweight compared to $26.5 \%$ of Italy [5]. The epidemic overweight/obesity in our region makes South Italy, particularly the Campania region, a privileged perspective to understand the correlation between HS, overweight/obesity and related conditions, such as insulin resistance, diabetes and metabolic syndrome.

Over recent years, several authors have tried to explore the link between obesity, metabolic syndrome and HS. Nowadays, overweight/obesity are considered to have a leading role in the pathogenesis of several diseases. Regarding HS, overweight/obesity seem to play a role on multiple aspects. First, overweight/obese individuals have more and larger skin folds, whereby skin-skin contact occurs, enhancing mechanical friction. Mechanical friction contributes to HS development by promoting follicular occlusion and by triggering rupture of dilated follicles in a genetically susceptible individual. The higher than usual skin temperature and humid microclimate in skin folds favor bacterial growth. Bacterial infection has long been suspected to play a secondary role in the pathogenesis of HS, as the bacterial colonization leads to a series of pathogen-associated molecular pathways, which may contribute to perpetuating and exacerbating local inflammation [6]. Third, obesity causes a low-grade, subacute, systemic inflammatory state. This inflammatory state produces increased circulating levels of pro-inflammatory cytokines, such as TNF- $\alpha$. TNF- $\alpha$ is a pro-inflammatory cytokine that plays an important role in the control of lipid metabolism and interferes with the signaling of insulin, whose circulating levels are increased with obesity and decreased with weight loss. An increase in TNF- $\alpha$ in skin lesions of patients with HS has been demonstrated and suggests a key role of this cytokine in the pathogenesis of HS [7].

In 2008, Revuz et al. [8] demonstrated a significant association between BMI and HS: the risk of developing HS increased by 1.12 for each increase of 1 BMI unit. When divided into BMI categories of normal weight, overweight and obese, obese patients had more severe HS than overweight patients, and overweight patients had more severe HS than normal-weight patients. Kromann et al. [9] investigated the effect of a substantial weight loss on disease severity as assessed by flares and number of involved regions. Patients were given a retrospective questionnaire more than 2 years after bariatric surgery to collect data on pre- and postsurgery HS symptoms and disease severity. The number of patients reporting HS symptoms after weight loss decreased by $35 \%$ and the mean number of involved sites was reduced from 1.93 to 1.22 following weight loss. Thomas et al. [10] and Boer [11] reported some cases which showed rapid improvement of longstanding treatment-refractory HS following bariatric surgical intervention and subsequent dramatic weight loss.

In 2012, Sabat et al. [12] showed for the first time that HS patients have a high prevalence of the metabolic syndrome. From then on, three more teams of scientists have confirmed these findings suggesting the importance of taking into account that HS patients may have one or more undiagnosed components of the metabolic syndrome despite their young age, and highlighting the need of an appropriate targeted screening. Although robust evidence is still lacking, treatment of HS and suppression of systemic inflammation could help reduce the incidence and severity of comorbidities. Anyway, early detection and treatment of these morbidities in patients with HS may prevent late complications, particularly cardiovascular disease. Lifestyle modifications, including weight loss and smoking cessation, might be necessary not only to decrease the severity of HS, but also to reduce the risk of arising of these comorbidities.

Our observations suggest that, like BMI, also HOMAIR and insulin secretion during oral glucose tolerance test correlate with HS severity. A recent paper published by our team has also demonstrated that mammalian target of rapamycin (mTOR) gene expression is increased in lesional as well as nonlesional skin of HS patients and this is statistically correlated with the severity of HS. Recent studies have established that increased activity of mTOR may lead to abnormal lipid metabolism and diabetes progression.

In conclusion, HS is burdened with numerous metabolic complications. Early detection and timely intervention have the potential to reduce HS complications. This may have implications for quality of life and health care costs. Unfortunately, the delay in diagnosis is frequent not only in South Italy, but all around the world: in a study conducted in 29 medical centers of 24 countries of all continents, the average diagnostic delay was 7.2 years [13]. Our data could be a very important tool to underline that patients affected by HS could obtain a correct diagnosis with much less delay with particular attention to metabolic alterations in adolescence. Their clinical management would be entrusted by multidisciplinary teams (dermatologist/endocrinologist/plastic surgeon) at an earlier time to ensure the best possible course of this disease. 


\section{Statement of Ethics}

The authors declare that their research complied with the guidelines for human studies and welfare regulation and the subjects participating in the research have given their informed consent.

\section{References}

1 Gold DA, Reeder VJ, Mahan MG, Hamzavi $\mathrm{IH}$ : The prevalence of metabolic syndrome in patients with hidradenitis suppurativa. J Am Acad Dermatol 2014;70:699-703.

2 Menter A: Recognizing and managing comorbidities and complications in hidradenitis suppurativa. Semin Cutan Med Surg 2014; 33(suppl 3):S54-S56.

3 Shalom G, Freud T, Harman-Boehm I, Polishchuk I, Cohen AD: Hidradenitis suppurativa and metabolic syndrome: a comparative cross-sectional study of 3,207 patients. Br J Dermatol 2015; 173:464-470.

4 Bettoli V, Naldi L, Cazzaniga S, Zauli S, Atzori L, Borghi A, Capezzera R, Caproni M, Cardinali C, De Vita V, Donini M, Fabbrocini G, Gimma A, Pasquinucci S, Patrizi A, Pinna AL, Raone B, Ricci M, Virgili A, Balestri R: Overweight, diabetes and disease duration influence clinical severity in hidradenitis suppurativa-acne inversa: evidence from the national Italian registry. Br J Dermatol 2016; 174:195-197.

5 Osservatorio Nazionale sulla salute nelle regioni italiane (National Observatory of Health in Italian Regions) (ed): Rapporto Osservasalute 2014 (Osservasalute Report 2014). 2015.

\section{Disclosure Statement}

All the authors declare that they do not have any possible conflicts of interest.
6 Matusiak L, Bieniek A, Szepietowski JC: Bacteriology of hidradenitis suppurativa - which antibiotics are the treatment of choice? Acta Derm Venereol 2014;94:699-702.

7 Mozeika E, Pilmane M, Nürnberg BM, Jemec GB: Tumour necrosis factor-alpha and matrix metalloproteinase-2 are expressed strongly in hidradenitis suppurativa. Acta Derm Venereol 2013;93:301-304.

8 Revuz JE, Canoui-Poitrine F, Wolkenstein P, Viallette C, Gabison G, Pouget F, Poli F, Faye O, Roujeau JC, Bonnelye G, Grob JJ, BastujiGarin S: Prevalence and factors associated with hidradenitis suppurativa: results from two case-control studies. J Am Acad Dermatol 2008;59:596-601.

9 Kromann CB, Ibler KS, Kristiansen VB, Jemec GB: The influence of body weight on the prevalence and severity of hidradenitis suppurativa. Acta Derm Venereol 2014;94:553557.

10 Thomas CL, Gordon KD, Mortimer PS: Rapid resolution of hidradenitis suppurativa after bariatric surgical intervention. Clin Exp Dermatol 2014;39:315-317.
11 Boer J: Resolution of hidradenitis suppurativa after weight loss by dietary measures, especially on frictional locations. J Eur Acad Dermatol Venereol 2016;30:895-896.

12 Sabat R, Chanwangpong A, Schneider-Burrus S, Metternich D, Kokolakis G, Kurek A, Philipp S, Uribe D, Wolk K, Sterry W: Increased prevalence of metabolic syndrome in patients with acne inversa. PLoS One 2012; 7:e31810.

13 Saunte DM, Boer J, Stratigos A, Szepietowski JC, Hamzavi I, Kim KH, Zarchi K, Antoniou C, Matusiak L, Lim HW, Williams M, Kwon HH, Gürer MA, Mammadova F, Kaminsky A, Prens E, van der Zee HH, Bettoli V, Zauli S, Hafner J, Lauchli S, French LE, Riad H, ElDomyati M, Abdel-Wahab H, Kirby B, Kelly G, Calderon P, Del Marmol V, Benhadou F, Revuz J, Zouboulis CC, Karagiannidis I, Sartorius K, Hagströmer L, McMeniman E, Ong N, Dolenc-Voljc M, Mokos ZB, Borradori L, Hunger RE, Sladden C, Scheinfeld N, Moftah N, Emtestam L, Lapins J, Doss N, Kurokawa I, Jemec GB: Diagnostic delay in hidradenitis suppurativa is a global problem. Br J Dermatol 2015;173:1546-1549. 